Itinéraires Itinéraires

Littérature, textes, cultures

\title{
Souci narratif et enjeux de mobilité : le récit de société à l'épreuve du reportage littéraire
}

Pascal Gin

\section{(2) OpenEdition}

\section{Journals}

Édition électronique

URL : http://journals.openedition.org/itineraires/2662

DOI : $10.4000 /$ itineraires.2662

ISSN : 2427-920X

Éditeur

Pléiade

Référence électronique

Pascal Gin, «Souci narratif et enjeux de mobilité : le récit de société à l'épreuve du reportage littéraire », Itinéraires [En ligne], 2015-1 | 2015, mis en ligne le 18 décembre 2015, consulté le 23 avril 2019. URL : http://journals.openedition.org/itineraires/2662 ; DOI : 10.4000/itineraires.2662

Ce document a été généré automatiquement le 23 avril 2019

\section{(c) (i) (9)}

Itinéraires est mis à disposition selon les termes de la licence Creative Commons Attribution - Pas d'Utilisation Commerciale - Pas de Modification 4.0 International. 


\title{
Souci narratif et enjeux de mobilité : le récit de société à l'épreuve du reportage littéraire
}

\author{
Pascal Gin
}

\section{Du souci narratif à l'écriture du social}

Que le social suscite aujourd'hui une pratique narrative accrue est un constat que posent plus d'une réflexion d'envergure sur la condition contemporaine. Certes, on peut s'attendre à ce que l'érosion heuristique des grands récits se double, au niveau des microénonciations, d'une hyperactivité symbolique, soit d'efforts renouvelés au quotidien pour configurer par le récit l'être et l'agir. Variant de la préoccupation sous-jacente à la pulsion pathologique, ce que l'on peut désigner du terme de souci narratif procéderait toutefois plus spécifiquement de conjonctures fortement mondialisées, de transformations que subit le sujet social comme du caractère inédit que présentent les situations de risque contemporaines. Pour les premières, on peut relever à titre d'exemple le scriptage translocal de récits de vie qu'analyse l'anthropologue Arjun Appadurai (1996), soit comment l'autoreprésentation de comportements pourtant des plus locaux intègre une diversité d'imaginaires mondialisés. La question du sujet et de ce qu'il devient dans le contexte de modernités tardives est, elle, largement étayée par la réflexion sociologique d'Anthony Giddens (1991), telle qu'elle concerne tout particulièrement l'interpellation réflexive d'un sujet sans cesse appelé à redéfinir visées et pratiques le confirmant dans la maîtrise d'un projet de vie. Quant aux situations de risque dont il retourne, celles-ci évoquent tout particulièrement la pensée d'Ulrich Beck, soit une sociologie attentive aux communautés de risque que fédère, dans un travail d'introspection narrative, l'autorité conflictuelle et déclinante des discours d'expertise et de savoir (Beck et Willms 2004). Telles seraient, selon les représentations épistémiques qu'en façonne la pensée du social, certaines des conjonctures motivant une individuation marquée de ce que l'on pourrait appeler le «souci narratif». C'est dire combien, dans 
cette perspective, production narrative et composition du social s'interpénètrent et résistent à l'effet de groupe de quelque récit dominant.

Il apparait dès lors légitime de se demander ce qu'il advient aujourd'hui des récits de société que produisent, par effet de retour narratif, les textualisations contemporaines, notamment celles associées aux pratiques littéraires. S'il est un fait que l'on ne saurait présumer d'une convergence par simple effet de reflet, il n'en demeure pas moins que, sensible tant à un retour du social en littérature qu'à des pratiques textuelles fragilisant postures et formes narratives établies, la critique s'accorde pour souligner un passage à des écritures du social tout aussi renouvelées que peut l'être, au regard de la sociologie, le monde en recomposition motivant justement le geste littéraire ${ }^{1}$. Le propos de la présente étude sera précisément de cerner de plus près ces rapprochements éventuels en s'enquérant des transformations que subit le récit de société lorsque le prend en charge l'écriture de presse, et plus spécifiquement le sous-genre du journalisme littéraire. Il s'agira de déterminer dans quelles mesures le souci narratif reconfigurant le référent société s'y actualise textuellement, sachant combien cette écriture peut se montrer contrainte quant à l'énonciation du social, ne jouissant pas a priori de la latitude énonciative dont se réclame la production littéraire.

3 Le corpus dont il va être question relève d'une forme narrative étroitement liée au genre du reportage, forme longue du récit journalistique qui se traduit par une textualité mixte, puisqu'elle mise tant sur une écriture professionnelle du réel, investie d'une fonction de témoignage public, que sur une écriture du sujet tirant parti de l'introspection autobiographique ou parfois même de la spéculation digressive de l'essai ${ }^{2}$. Adapté de l'anglais, le syntagme "journalisme littéraire " met en mémoire terminologique ces tensions constitutives du genre, soit une distanciation par divers biais littéraires à l'égard du discours de presse que norme l'axiologie d'un rapport au réel fondé sur le présupposé d'une immédiateté objectivante. Cette distanciation couvre des amplitudes inégales comme en attestent les deux textes retenus. Ainsi le reportage que la journaliste Florence Aubenas consacrait en 2010 à la précarisation de l'emploi en France, paru sous le titre Le Quai de Ouistreham, relève-t-il davantage d'une variante littéraire que d'une transgression du discours de presse. En revanche, le livre Terminal Frigo publié par Jean Rolin en 2005, récit documentant une série de déplacements effectués par ce journaliste français dans diverses installations portuaires du littoral français, sera ici invoqué à titre de contrepoint exploitant plus radicalement l'écart littéraire.

\section{Journalisme littéraire et récriture du social}

4 La délégitimation du discours de presse et, partant, des récits de société qu'il met en circulation fonde la légitimité même du journalisme littéraire. L'écriture dont celui-ci s'acquitte procède effectivement d'une complexification des conditions d'énonciation ou de mise en texte qui lui confère spécificité et pertinence génériques. Écrire l'actuel sans subir les contraintes formelles du genre bref, épaissir le rapport au réel dans l'épreuve biographique du vécu et l'élargissement des prises de parole sont, parmi d'autres, autant d'écarts dont peut mais doit aussi se prévaloir cet autre journalisme pour se ménager, à la périphérie de l'information de masse, une marge de reformulation. Cette manœuvre garantissant un positionnement marginal mais privilégié dans le champ de la presse écrite peut de fait se décliner en véritables topoï narratifs embrayant la réénonciation du réel social. Ainsi dans l'avant-propos de ce «reportage" qu'elle consacre à la 
précarisation de l'emploi en France, la journaliste Florence Aubenas biographise-t-elle les carences du discours médiatique face à un état de société (nous sommes en 2008) que résume, sans prise aucune sur le tangible des faits, le récit-type de la «crise». Le sentiment de mise en échec professionnel va explicitement motiver une volonté de dépassement du déjà dit journalistique et du récit de société auquel il se borne. À la "crise ", sorte de sommaire narratif réitéré à volonté, va alors se substituer le récit fortement localisé d'une immersion individuelle (Florence Aubenas, demandeuse d'emploi) dans l'espace circonscrit du réel social. Chez Jean Rolin, le rapport à l'énoncé de presse et à la mise en récit du fait social dont il s'acquitte s'apparente certes à une autre posture. Le narrateur ne décline pas, dans l'avant-texte d'un avant-propos, l'ethos professionnel du journaliste soucieux de recouvrer la pleine extension d'une parole narrative. Son « livre sur les ports » n'en témoigne pas moins d'une contre-énonciation prenant régulièrement le pas sur le discours de presse, à la faveur d'articles de journaux fréquemment cités, régulièrement commentés, souvent concurrencés par les récits de terrain que coordonne Terminal Frigo.

5 Or, quand bien même le journalisme littéraire conteste la légitimité des récits à forte écoute ou de grande lisibilité qui configurent l'ordre et la cohérence des faits sociaux, il n'en contribue pas moins à les perpétuer par la fonction même qui lui revient. Il s'agit de narrer mieux, autrement, en situation de récit rapproché mais sans perdre de vue l'horizon collectif, national très souvent, institutionnel parfois, des faits remis en récit. Ainsi la tradition du journalisme de simulation à laquelle se rattache Le Quai de Ouistreham présuppose-t-elle des clivages structurels, une marginalité à infiltrer, des substitutions identitaires à effectuer selon des codes établis pour qu'un extérieur puisse prendre conscience d'une division interne, des classes ou regroupements, conditions ou pratiques que définit la précarité de l'emploi dans une société en état de crise ${ }^{3}$. Le «faisant ceci, je fais cela» du journaliste infiltré cultive donc chez le lecteur de reportage l'attente d'énoncés qui documentent les marges et par le fait même entérinent la composition clivée mais totalisable d'un espace de représentation du social. De forme beaucoup plus lâche, le récit de Jean Rolin se soustrait davantage pour sa part aux pratiques établies susceptibles d'inscrire les faits relatés dans une matrice narrative leur conférant d'emblée forme sociale. Le repli narratif sur le social concernerait davantage, dans cet autre texte, la figure narrative du reporter aventurier, mettant à profit son autonomie de déplacement pour prendre le risque d'une transgression des lieux convenus de l'espace public, pour introduire donc la lecture dans des sites attestant de socialités limitrophes (celles de l'immigration clandestine, du vagabondage urbain) dont on ne tire pas moins des régularités.

6 Synthétisant cette double contrainte, l'on peut ainsi parler d'une pragmatique énonciative liant intimement le journalisme littéraire à un récit de société qu'il s'agit de soustraire, mais pour en produire une autre formulation, à l'hégémonie du discours de presse.

\section{Récits de société : entre persistance et fragilisation}

7 Du journalisme d'infiltration d'Aubenas à l'enquête sans contours particulièrement définis de Rolin, le récit de société, ou du moins ce qui s'y apparente le plus manifestement, parait subir un déplacement marqué. Sollicité par le hors-texte de ce qui s'impose à l'écriture tel un fait social déjà posé, Le Quai de Ouistreham configure 
précarisation de l'emploi, conditions de travail, exclusion et déclassement dans un récit progressant vers l'effet de groupe d'une expérience collective. D'agences d'intérim en équipes de travail, la sériation des péripéties tissera effectivement des régularités conférant au récit de société une validation tout inductive. Quelque individués qu'ils soient, rencontres, embauches et incessants déplacements tirent cohérence de l'effet de dramatisation d'une littérature des types, en lesquels s'incarne donc l'espèce de comportements établis. Le Quai de Ouistreham atteste en cela de persistances du récit de société, soit du réinvestissement dans un mode d'intelligence narrative susceptible de rendre vraisemblable des mutations du social en rupture pressentie de représentation. Il en va toutefois tout autrement du texte de Jean Rolin, qui réclame de ce fait une lecture plus minutieuse.

8 Certes le récit épousera pour une large part les contours d'une géographie physique circonscrivant une entité nationale sur le pourtour de son littoral. Ce récit se montre toutefois par trop sensible aux porosités frontalières (tout particulièrement à l'immigration clandestine et aux flux médiatiques) pour que l'on puisse invoquer la cohésion de quelque tour de France ou récit de la nation. Ce serait au niveau du travail de composition dont s'acquittent les alternances topographiques d'un lieu à l'autre que seraient à localiser les principales résonances sociales. La progression géographique des lieux visités se prête effectivement à un agencement intercalant des lieux de transit fortement associés à des collectivités migrantes et des lieux de résidence couplés à la figure collective du résident de longue date. Or s'opère à la faveur de cette progression contrastée une jonction entre le social et le géographique, de sorte qu'une socialité du lieu nous met de fait sur la voie de véritables récits de société. Ceux-ci mobilisent alors le texte sous formes de récits de parole, énonciation rapportée traçant les contours du social tels que se les représentent les sujets concernés ${ }^{4}$.

9 Les récits ainsi consignés s'avèrent pour le moins contrastés. Alors que le texte s'attarde sur le Bureau central de la main-d'œuvre à Dunkerque (ou BCMO, bâtiment phare du mouvement syndical, condamné à la démolition), sollicitant les témoignages sur l'essor, sur l'apogée et sur le déclin du syndicalisme ouvrier, le narrateur va se faire le rapporteur d'un pathos nostalgique, d'un discours du ressentiment, de l'essentialisme factionnaire des appartenances et désappartenances politiques. Des propos et jugements recueillis émerge une seconde dimension frontalière du littoral. Celle-ci concerne, au point de jonction entre l'économie nationale et le commerce international, les structures internes de socialisation qui transforment transits maritimes et mobilités marchandes en un espace domestique, soit celui des dockers, de leur quotidien, tant professionnel qu'individuel et familial. De la rumeur sociale se dégage très nettement la référence à une territorialité révolue. L'emprise du syndicalisme sur le quotidien ouvrier des années 1960 et 1970 se traduisait effectivement par une sédentarité investissant l'espace vécu: passage obligé par le BCMO, lieu de travail régi par un principe de filiation (ce «[...] caractère héréditaire, dynastique, du métier de docker [...]», Rolin $2005: 74$ ), autorité syndicale exerçant son influence politique jusque sur l'aménagement municipal, communauté urbaine soudée autour des installations portuaires ${ }^{5}$. Territorialisation donc fortement localisée que vont progressivement remettre en cause tensions et crise économiques (un « [...] déclin apparemment irréversible face à la concurrence des autres ports européens [...]», Rolin 2005: 83-84), l'incapacité structurelle de s'adapter à l'intensification comme à la diversification des flux mondialisés. Or, à proximité même de lieux subissant désindustrialisation et détraditionnalisation, l'énonciation du journaliste 
va croiser, relayer et configurer des énoncés se référant à un tout autre hors-texte sociétal, soit à des pratiques diasporiques et à l'espace social qu'elles redistribuent. Site industriel que traversent les dynamiques d'une mondialisation économique, le chantier naval se fait ainsi le lieu d'un récit de société en contre-énonciation. Terminal Frigo va tracer sur ce même littoral portuaire les contours d'une géographie de groupe propre à la présence tout au plus contractuelle d'une main-d'œuvre étrangère. Ingénieurs et ouvriers en l'occurrence indiens n'en impriment pas moins sur le sol français un mode d'organisation collective dont le texte tirera un tout autre récit en croisant témoignages et propos rapportés. Le récit de société se déploie alors tel un réseau sur lequel figurent des zones investies d'une forte pertinence ontologique (lieux d'origine, auxquels on retourne pour conférer légitimité aux pratiques matrimoniales), des embranchements que dynamisent des potentiels migratoires accrus ou perçus comme tels (établissement aux États-Unis), des parcours privilégiés faisant se recouper pratiques familiales et déplacements touristiques (dans telle ou telle région de la diaspora) ${ }^{6}$. Le social dont il est alors fait récit consiste précisément à maintenir, dans l'extension d'un espace vécu élargi, les relais d'une cohérence collective.

10 À cette diversification des récits de société va venir s'ajouter un effet de côtoiement sapant ou du moins réduisant leur légitimité respective. La totalisation vers laquelle ils tendent à titre de récit imposant la cohérence d'un ordre de représentation collectif se voit fragilisée par leur contiguïté réciproque, tel récit pouvant difficilement se soustraire à l'ombre que lui porte par défaut telle autre énonciation narrative du social. Distribués entre la communauté des travailleurs indiens et les syndicalistes ou bénévoles français leur apportant soutien, ces récits suscitent des incompréhensions mutuelles au contact d'axiologies parfois peu conciliables, témoignant de frontières discursives particulièrement étanches. On relève, chez les premiers, la valeur de l'effort ou une éthique du travail s'accommodant avec méfiance ou incrédulité de la sollicitude syndicale, la norme de l'ethos collectif soucieuse de la rumeur publique ${ }^{7}$, une désapprobation ouverte à l'égard de l'égalité entre les sexes ou encore d'un laxisme associé aux pratiques religieuses ${ }^{8}$. À l'inverse, les propos européens s'acquittent d'un tiers-mondisme dépréciateur, d'un orientalisme se repliant sur l'encyclopédisme culturel ou le référent historico-médiatique, voire d'un universalisme syndicaliste de la cause commune ${ }^{9}$. Récits de parole relevés à la faveur d'incessants déplacements, ces récits de société ou plus exactement les fragments qui s'y rapportent s'imposent donc au journaliste de terrain tant par la force de leur assertion que par leur mutuelle fragilisation. S'il est à ce titre un récit de société qu'organise à son tour Terminal Frigo par ce qu'il série dans sa progression narrative, celui-ci mettrait dès lors en valeur une délégitimation de l'autorité totalisante à laquelle peuvent prétendre, non pas de simples rémanences narratives, mais des récits en situation d'empiétements énonciatifs que l'on ne mobilise pas moins, voire davantage. Se soustrayant à l'impératif d'une substitution narrative perpétuant tel ou tel récit de société comme unique expression de l'expérience collective, cet autre journalisme littéraire semble donc exploiter une marge d'énonciation novatrice, situant par le fait même l'écriture du social en rapport d'homologie partielle avec certaines mutations contemporaines dont il se fait écho narratif ${ }^{10}$. 


\section{Récits de parcours et enjeux de mobilité}

11 Quelque déterminantes qu'elles soient, les incursions sous forme de commentaires narratifs dans le détail de l'énonciation collective n'en demeurent pas moins épisodiques à l'échelle d'un texte que n'oriente pas l'exclusivité d'un phénomène d'actualité clairement circonscrit. Aussi de tels récits de société et les recompositions par apposition qu'ils subissent ne suffisent-ils pas à circonscrire dans ce reportage aux marges du reportage une écriture $\mathrm{du}$ social dont il faut alors situer ailleurs les principales émergences textuelles. Récit, Terminal Frigo l'est avant tout à la faveur d'un principe de progression narrative faisant coïncider énonciation journalistique et le chronotope de la route $^{11}$. De Dunkerque à Calais, de Royan à Sète, le récit compose par fragments, sur un axe nord-sud, un réseau toponymique que ne légitime aucun itinéraire, que ne préfigure jamais l'espace projeté de la destination. Pour l'essentiel, l'espace textuellement produit fait donc ainsi entorse à l'unité intentionnelle du récit de voyage. Certes, ce principe de progression narrative peut être pris en charge depuis les pratiques mêmes du journalisme littéraire. Aller au fait, ce que l'on évoque comme une démarche immersive, n'est pas une simple catachrèse évoquant l'écart que cherche à écrire cet autre journalisme. Le rapport renouvelé au fait s'éprouve sur un mode topographique, dans un parcours d'espace le long duquel se déploie, consubstantiellement, l'expérience du fait. Au sein même du registre journalistique, on pourrait évoquer à ce propos un recours au récit de parcours, stratégie consistant à produire une biographie des déplacements susceptibles de narrativiser tel ou tel témoignage ${ }^{12}$.

12 L'intensification de ces récits de parcours dans Terminal Frigo atteste toutefois d'une mobilisation autre que générique. Ce type de récit s'extrapole effectivement à toutes sortes de mobilités, le texte déclinant une multiplicité de parcours, dont celui du narrateur, que tracent sur le paysage frontalier nomadisme urbain et pratique diasporique, mais encore immigration clandestine, trafic industriel et circulation des signes médiatiques ${ }^{13}$. À distance de l'encadrement de quelque unique récit de société, le texte fait ainsi se coïncider une situation d'énonciation et des cadres de représentation saturés par la référence au déplacement. Il y a lieu à ce propos de faire valoir la plausibilité de corrélations entre production textuelle et sociologie de la mobilité ${ }^{14}$. Sans qu'il puisse être ici question de détailler les principales interventions théoriques contribuant à cette orientation de la réflexion sur le contemporain, il convient de souligner combien les mobilités y interviennent à titre de pratiques paradigmatiques structurant aujourd'hui l'activité sociale, actualisant selon les formes qu'elles revêtent inégalités marquées et fort potentiel de différentiation, mais surtout relayant dans l'espace circonscrit de situations locales flux et processus de mondialisation, soit la dynamique même qui modifie sans doute plus qu'aucune autre l'ordre du social contemporain ${ }^{15}$.

Que le récit de parcours puisse participer chez Jean Rolin d'une écriture du social n'est pas un effet de lecture qu'informe uniquement la transversalité du regard sociologique. Par ses effets de composition, Terminal Frigo ne cesse de mettre en texte, leur conférant sens, des mobilités référentielles signifiées dans leur disparité d'usage ou de statut mais aussi dans le maillage collectif qu'elles rendent possible. Ce sera ainsi l'écart entre les expériences clandestine et touristique d'une traversée de la Manche que le récit de parcours détaillera ${ }^{16}$. Ce seront également les transits anthropologiques et strictement 
matériels que ces mêmes récits juxtaposeront, attestant ce faisant d'une co-pénétration des géographies humaines et non humaines, l'itinéraire du conteneur maritime ${ }^{17}$ croisant les vagabondages péri-urbains du sans-abri ${ }^{18}$. Ce seront surtout les effets d'étoilement social des pratiques de mobilité auxquels le récit de parcours se montrera sensible, détaillant ici comment la maîtrise technologique de la mobilité des signes (caméscope, téléphone, Internet) s'intègre dans les pratiques communautaires de travailleurs étrangers en exil temporaire ${ }^{19}$, relevant là des nœuds de mobilité entrecroisant trajets clandestins et itinéraires urbains de l'assistance associative ${ }^{20}$.

Il paraît opportun à ce stade de revenir sur ces récits de société que le texte situe en rapport de co-énonciation conflictuelle. La mutuelle interception de leur légitimité respective accède précisément au texte par un biais narratif qui n'est pas en dernière analyse discours de parole, mais bel et bien discours d'événement, récit de parcours. Les énoncés à valeur collective sur lesquels s'arrête le texte procèdent justement d'une conjoncture que structurent les déplacements qui s'y tracent et s'y organisent. Les mobilités humaines de ces travailleurs indiens qui alternent mission ou contrat en Europe et au Moyen-Orient, immigrent pour certains au sein d'une communauté établie à l'étranger, pratiquent à intervalles réguliers des retours sur les lieux de l'origine, l'ensemble donc de ces déplacements humains sont de fait assujettis à la mobilité de savoirs et savoir-faire techniques justifiant que l'on dépêche d'un continent à l'autre une main-d'œuvre souvent très qualifiée. En retour, l'exploitation de cette même maind'œuvre dépend d'une circulation monétaire, investissements, dépenses salariales et transactions internes aux économies de la diaspora. Or le flux mutuellement consolidé de ces mobilités va soudainement s'inverser alors que se tarissent les apports financiers du fait de la faillite imminente affligeant la multinationale concernée. Cet état d'inertie qu'introduit la perturbation des flux de capitaux se propage alors, tel qu'en rend compte le récit, à l'ensemble du réseau de mobilités ${ }^{21}$. À la fluidité succède une déstabilisation immobilisant la collectivité des travailleurs dont le statut sur le territoire étranger devient des plus problématiques ${ }^{22}$. L'onde de choc se répercutant sur ce que l'on peut appeler, dans la perspective théorique que défriche John Urry (2007), l'intégralité d'un mobility system dévoile de profondes ruptures, qui s'affirment alors que s'accentuent les contacts entre cette présence soudainement exogène et diverses collectivités ou instances endogènes. Le récit de société contrarié n'intervient en ce sens dans Terminal Frigo qu'à titre de complication que le texte localise sur la trame d'un récit de parcours particulièrement étoffé, pour le moins mondialisé, où le social se produit dans l'interaction complexe de mobilités fluctuantes. En d'autres termes, le récit de parcours et les enjeux de mobilité qu'il série priment de fait le récit de société.

Il importe de constater à ce propos que même au sein de ce journalisme de simulation minimisant pourtant chez Florence Aubenas les transgressions littéraires du journalisme de presse, le récit de parcours va lui aussi venir à intervalles réguliers déplacer la mise en texte du social. Dominera alors, d'entrevues en lieux de placement, le rythme collectif d'un récit en constants trajets. Rythme que doublera par ailleurs un commentaire liant accès à l'emploi, condition d'automobilité et gestion du transport individuel. Rythme qu'axiologisera également une intervention paratextuelle dédicaçant le reportage à la mémoire du véhicule de fortune dont la journaliste aura fait le simulacre d'acquisition. Les quelques passages suivants donnent un bon aperçu de la fréquence du recours au récit de parcours, que celui-ci intervienne en discours rapporté ou en discours direct, sous le mode du témoignage ou de la simulation : 
Vers $10 \mathrm{~h} \mathrm{45,} \mathrm{le} \mathrm{jour} \mathrm{de} \mathrm{mon} \mathrm{inscription,} \mathrm{nous} \mathrm{sommes} \mathrm{une} \mathrm{quinzaine} \mathrm{à} \mathrm{attendre}$ dans un des huit sites Pôle Emploi de Caen [...]. L'essentiel de la discussion tourne autour de la meilleure manière de me rendre au premier rendez-vous d'orientation dans une antenne différente, à l'autre bout de Caen. (Aubenas $2010: 25-26$ )

Je demande le chemin pour le Novotel, où se tient le Salon pour l'emploi [...] j'ai changé de quartier. Je remonte maintenant le long de rues toutes droites, plantées de maisons récentes, au toit pointu. Puis, les immeubles deviennent de plus en plus hauts, les voitures roulent de plus en plus vite. Quand on arrive près de l'échangeur d'autoroute, dans la zone industrielle, on y est. (Aubenas 2010 : 33-34)

C'est le tout petit matin. La veille, pour être sûre de ne pas arriver en retard, j'ai fait deux fois le trajet avec le Tracteur, ma nouvelle voiture. Le rendez-vous est à $5 \mathrm{~h} 30$, au port d'embarquement du ferry-boat, pour la matinée de formation. (Aubenas $2010: 74)$

Arriver jusqu'au ferry est un nouveau périple. Il faut pénétrer dans la zone sous douane en montrant un badge avec une photo, fourni par la société. Parfois, des vigiles sortent de la guérite et s'accroupissent pour ausculter les essieux ou les

habitacles, en parlant de trafics et de clandestins. (Aubenas $2010: 76$ )

Le texte pratiquera même à l'occasion une permutation narrative indexant précisément l'écriture du social sur le récit de mobilité. Figure par excellence de l'abstraction du collectif, la foule en mouvement lors d'une « marche contre la crise » coordonne le topos de la rue et une crise socio-économique en quête de référent. Or, immergée en situation de bain de foule, la journaliste séquentialisera un mouvement physique évoluant vers sa dissolution, inapte à s'acquitter de toute énonciation collective «[...] sans colère, sans slogan réel, comme si sa seule revendication était sa propre masse» (Rolin, 2005 : 56). Départ impromptu, problème d'orientation, participation tout au plus épisodique, pause quasi touristique disent dans une commune parole la lassitude de parcours auxquels le mouvement social, ici des plus littéraux, ne confère aucune unitée ${ }^{23}$. Le texte ira jusqu'à faire intervenir un récit de vie assumé par une syndicaliste retraitée, qui servira de contrepoint historique à la jonction du social et des pratiques de mobilité. Le parcours révolu ainsi évoqué tracera la ligne droite d'un trajet générationnel, du monde rural au premier relais urbain «en suivant la ligne de chemin de fer », itinéraire physique que dédoubleront l'émergence d'une conscience de classe et la socialisation dans le mouvement syndical. L'unidirectionnalité, la prédictibilité, l'irréversibilité de cet itinéraire aujourd'hui révolu feront alors pendant à l'errance collective s'épuisant dans le contemporain d'une « marche pour la crise ${ }^{24} »$.

\section{Des récits de parcours aux récits de soi}

17 Peut-on dès lors citer l'expérience collective des mobilités à titre de refocalisation référentielle du social? On ne saurait de fait cautionner sans réserve une telle lecture de l'écriture du social dans Terminal Frigo, récit au trajet de prime abord incertain. Certes, consignant le détail des dates et lieux, documentant sources et propos recueillis, le texte s'acquitte d'une démarche journalistique sensible aux mouvements et déplacements qui structurent aujourd'hui sentiments d'appartenance et situation collectives. Force nous est toutefois de constater que la maîtrise journalistique du réel à énoncer faiblit chemin faisant. La constance du récit de parcours sur lequel elle s'exerce croise effectivement la réflexivité non moins soutenue d'un autorécit fragilisant la pratique du journalisme. On relève ainsi à longueur de texte des dérapages autobiographiques ${ }^{25}$, des digressions d'enquêtes sur des sujets très secondaires amorcées comme par réflexe à la faveur de tel ou tel détour ${ }^{26}$, l'absence répétée d'analyse concluante, des reprises d'investigation 
cédant au doute ${ }^{27}$, comme si donc la prolifération des déplacements et bifurcations saccadant le parcours du journaliste imprimait sa logique d'alternatives et de constants réamorçages à l'énonciation journalistique du réel. Le récit de parcours qu'assume un journalisme littéraire et la mesure du monde qu'il ambitionne s'infléchit alors dans le sens d'un récit de soi, récit contrarié d'une compétence de mobilité ne parvenant pas à se stabiliser dans des énoncés ayant valeur d'expertise, susceptibles d'imprimer un ordre cognitif à l'actualité. Or, plus résolument performative, cette autre modalité narrative du texte inscrit une autre modalité sociale dans l'énonciation du récit de parcours que celuici pratique. Il y a lieu ici de revenir, parce que le texte nous y conduit, sur les acquis d'une sociologie de la modernité avancée, dans la lignée des travaux d'Ulrich Beck sur la réflexivité individuée des situations de risque, ou ceux d'Anthony Giddens sur la maîtrise d'un projet narratif du soi, acquis dont on peut dire synthétiquement qu'ils dégagent des relations structurantes entre transformations du social et configurations narratives de l'individu ${ }^{28}$. Alors que les processus de modernisation s'orientent vers une hybridité structurelle débordant largement les cadres institutionnels ou territoriaux d'une première modernité, l'individu par une détraditionnalisation concomitante s'affranchit de tutelles collectives (appartenance de classes, figures de l'identitaire national, effet de groupe des métarécits), s'autoréalise ou se singularise en s'assumant et s'autonarrant comme projet de cette modernisation seconde. Il en ressort, terme de l'analyse, que la mise en intrigue des mobilités n'impose pas au texte l'effet de clôture narrative d'une nouvelle mesure du social. Écrivant le texte à distance du récit de société, le récit de parcours renforce de fait une expression du social en prise directe sur les enjeux biographiques du souci narratif, préoccupation fonctionnelle liant l'autonarration et un travail de rééquilibrage au quotidien, dont les modalités seraient celles de la précarité, de la récursivité, d'une instabilité routinière ${ }^{29}$. Si le journalisme littéraire présente une pertinence pour l'étude et la compréhension de l'énonciation du social contemporain, c'est aussi à titre de discours d'expertise, pratique de l'information susceptible de documenter le monde, s'inversant toutefois dans l'autoréflexivité d'un souci narratif sondant la précarité énonciative de son propos.

18 C'est sans doute dans l'entrecroisement des récits de mobilité et des récits de soi que le reportage de Florence Aubenas rejoint davantage encore l'écriture du social dégagée du texte de Jean Rolin. La mobilité s'inscrit alors, par personnage interposé, dans l'activité narrative d'un sujet que confrontent des exigences de déplacement, la gestion d'une mise à disponibilité et la maîtrise de l'espacement qu'elle réclame. Certains moments biographiques relatés vont ainsi sérier la maîtrise aléatoire d'un trajet ${ }^{30}$, une capacité de déplacement limité que contre toutefois un projet de vie dominant les ressources de l'automobilitée ${ }^{31}$, enfin un moment de réflexion mettant en rivalité un déplacement ferroviaire vers une autre ville pour prendre connaissance des motifs d'un licenciement et le déplacement quotidien que réclame localement un contrat temporaire ${ }^{32}$. Ce qui se communique ainsi de la journaliste-témoin au témoignage qu'elle recueille, c'est le récit d'enjeux de déplacement, des savoir-faire qu'ils mobilisent, et de la tension entre vouloirêtre-là et pouvoir-être-ici à gérer au quotidien. Le récit de soi se profile telle l'incessante réénonciation d'un projet d'autoréalisation exigeant du sujet qu'il maîtrise et réajuste trajets et connexité. L'écriture journalistique se fait donc le relais textuel d'une "condition biographique" (pour reprendre la formule pour le moins appropriée de Christine Delory-Momberger 2009) sous condition de mobilité saisie dans le détail de sa production narrative. 
C'est dire que le social ne s'énonce pas strictement dans les termes du regroupement, par le truchement d'une abstraction totalisante, classe ou communauté. Il s'éprouve davantage sur la courte durée, selon des modalités d'association mettant très concrètement des usagers en rapport avec la matérialité de dispositifs de mise en circulation, telle cette automobile d'occasion qui rendra possible un covoiturage, pratiques de spatialisation créant pour un temps du social. Le journalisme de simulation n'en est toutefois pas un de dissimulation. L'ethos journalistique de la lucidité référentielle requiert que soit régulièrement rappelé l'écart simulatif : « D'où je sors pour ne pas savoir que c'est normal?» (Aubenas 2010: 83). Loin toutefois d'atténuer la signification sociale du récit de soi, ces brefs rappels à la situation de simulation en élargissent de fait la portée. De la contrainte au choix, de l'évidente nécessité à la discrimination entre différents cours de vie, l'écart qui se creuse brièvement révèle le présupposé d'une autre autonarration, celle de la journaliste, qui assume l'exercice de la mobilité dans les termes d'une tout autre réalisation de soi, telle cette journaliste autobiographe d'inédits déplacements professionnels. Par le biais de cette mise à plat comparée des récits de soi, l'écriture de simulation fragilise l'aplomb de sa propre identité narrative. Elle montre par réfraction, ou plus exactement ironise par métalepse, une activité journalistique tout autant concernée par des pratiques de mobilités interpellant le sujet social dans son activité d'autonarration. D'un journalisme à l'autre, se répète alors la performance énonciative d'un tout autre récit de société.

\title{
Journalisme littéraire et souci narratif
}

\author{
Sans y avoir pris garde, j'ai changé de quartier. Je remonte maintenant le long de \\ rues toutes droites, plantées de maisons récentes, au toit pointu... Quand on arrive \\ près de l'échangeur d'autoroute, dans la zone industrielle, on y est. (Aubenas 2010 : \\ 33-34) \\ La chaleur a atteint son paroxysme au début de l'après-midi alors que je pédalais \\ sur la D1, entre la cimenterie et l'usine de polymères... (Rolin 2005 : 67)
}

Tirer récit, à la première personne, d'un déplacement urbain n'est sans doute pas sans conforter habitudes et attentes de lecture. De telles amorces narratives embraient certes, si l'on songe au seul xixe siècle français, sur des traditions romanesques des plus consacrées : Milord progressant dans la rue de l'Université de La Cousine Bette chez Balzac, tombereaux en enfilades de Nanterre au pont de Neuilly dans Le Ventre de Paris de Zola... De premières pages en débuts de chapitres, le mouvement citadin va toutefois se déployer très nettement chez Rolin comme chez Aubenas vers une tout autre mise en texte, récit de parcours et récits de soi primant en le recomposant une socialité du texte soustraite à l'intelligibilité narrative en tableau du récit de société. Si c'est là un constat que la critique contemporaine a su pleinement poser à l'égard d'écritures dites néoréalistes renouvelant le rapport littéraire au social ${ }^{33}$ - l'on pense ici à des auteurs tels que François Bon ou Annie Ernaux - le cas plus marginalisé de l'écriture de presse suscite rarement comparable intérêt, quand bien même la littérature française $\mathrm{du} \mathrm{xxI}$ siècle emprunte très souvent la voie de la non-fiction que fraie le journalisme littéraire ${ }^{34}$. Quelque ciblée qu'elle soit, l'étude de cas ici proposée suggère précisément la pertinence d'élargir analyse et compréhension de l'énonciation collective contemporaine à ces autres écritures du social. Si Bruno Latour (2006: 177-203) souligne l'importance d'une production du savoir social sachant oser des "comptes rendus risqués", se montrant davantage sensible, à l'encontre des grands récits de la sociologie, aux infralangages des 
sujets sociaux, on évoquera en conclusion combien les réaménagements du récit de société concernent tout autant les ambivalences de nos métalangages, dont ce journalisme littéraire se fait en quelque sorte l'énonciation rapportée, discours d'expertise aux prises avec la réflexivité d'un souci narratif qu'il performe tout autant qu'il l'énonce.

\section{BIBLIOGRAPHIE}

Adey, Peter, 2010, Mobility, Londres, Routledge.

Angenot, Marc, 1992, «Que peut la littérature ? Sociocritique littéraire et critique du discours social », dans J. Neefs et M.-C. Ropars (dir.), La Politique du texte. Enjeux sociocritiques, Lille, Presses universitaires de Lille, p. 9-27.

Aubenas, Florence, 2010, Le Quai de Ouistreham, Paris, Seuil.

Appadurai, Arjun, 1996, Modernity at Large: cultural dimensions of globalization, Minneapolis, University of Minnesota Press.

Bakhtine, Mikaïl, [1975] 2006, Esthétique et théorie du roman, Paris, Gallimard.

Barrère, Anne et Martuccelli, Danilo, 2005, « La modernité et l'imaginaire de la mobilité : inflexion contemporaine ", Cahiers internationaux de sociologie, vol. 1, n 1, p. 55-79.

Beck, Ulrich, 2003, La Société du risque : sur la voie d'une autre modernité, trad. de l'allemand, Paris, Flammarion.

Beck, Ulrich et Willms, Johannes, 2004, Conversations with Ulrich Beck, Cambridge, Polity Press.

Blanckeman, Bruno, 2002, Les Fictions singulières : étude sur le roman français contemporain, Paris, Prétexte éditeur.

-, 2005, « Le souci de société (sur quelques écritures néoréalistes) », dans M. Collomb (dir.), L'Empreinte du social dans le roman depuis 1980, Montpellier, Publications de l'Université Paul Valéry, p. 25-34.

Boucharenc, Myriam et Deluche, Joëlle (dir.), 2001, Littérature et reportage, Limoges, PULIM. Cresswell, Tim, 2006, On the Move: Mobility in the Modern Western World, New York, Routledge. Delory-Momberger, Christine, 2009, La Condition biographique. Essais sur le récit de soi dans la modernité avancée, Paris, Téraèdre.

Giddens, Anthony, 1991, Modernity and Self-Identity: Self and Society in the Late Modern Age, Stanford, Stanford University Press.

-, 2000, Runaway World: How Globalization is Reshaping our Lives, New York, Routledge.

Griffin, John, 1961, Black Like Me, Boston, Houghton Mifflin.

Hartsock, John, 2000, A History of American Literary Journalism: The Emergence of a Modern Narrative Form, Amherst, University of Massachusetts.

Kaufmann, Vincent, 2002, Re-Thinking Mobility: Contemporary Sociology, Aldershot, Ashgate. 
Lallemand, Alain, 2011, Journalisme narratif en pratique, Bruxelles, De Boeck.

Latour, Bruno, 2006, Changer de société, refaire de la sociologie, Paris, La Découverte.

Rolin, Jean, 2005, Terminal Frigo, Paris, POL.

Thérenty, Marie-Ève, 2007, La Littérature au quotidien. Poétiques journalistiques au XIX ${ }^{e}$ siècle, Paris, Seuil.

Urry, John, 2007, Mobilities, Cambridge, Polity Press.

Viart, Dominique et Vercier, Bruno, 2005, La Littérature française au présent : héritage, modernité, mutations, Paris, Bordas.

Wallraff, Günter, 2013, Tête de turc, trad. de l'allemand, Paris, La Découverte.

\section{NOTES}

1. Voir notamment les analyses de Dominique Viart (Viart et Vercier 2005) et Bruno Blanckeman (2002).

2. Pour une perspective d'ensemble, variant périodes et traditions nationales, on se référera aux ouvrages de John Harstock (2000), Marie-Ève Thérenty (2007) ou encore au collectif co-dirigé par Myriam Boucharenc et Joëlle Deluche (2001).

3. Parmi les reportages littéraires se rattachant à cette tradition, on consultera notamment ceux, ayant fait date, de John Griffin (1961) et Günter Wallraff (2013).

4. Nous sommes proches à ce propos de cette littérature qui ne "connaît qu'au second degré » selon l'expression de Marc Angenot (1992: 16), soit par réénonciation des discours qui la précèdent et l'entourent.

5. «[...] leur vrai patron, celui qui règle tous les détails de leur vie professionnelle et leur prodigue sans compter ses bienfaits [...] c'est le syndicat [...]. Réélu quatre fois à la mairie de Cappelle-la-Grande, Roger Gouvart [haut dirigeant syndicaliste de l'époque] y exerce apparemment une sorte de despotisme éclairé [...] "Ici, commente-t-il, il y a vingt ans, c'était tout des champs. J’y ai construit une nouvelle ville”.» (Rolin 2005 : 82 et 109-110, notamment)

6. Voir les sections du texte consacrées au personnage de Sunny Paul, travailleur indien « [...] âgé de vingt-neuf ans. Il en avait un de moins lorsqu'il est arrivé à Saint-Nazaire, avant même que n'y soit découpée la première tôle du Queen Mary 2. Originaire de Trichur, dans l'État de Kerala, il appartient à la minorité catholique, il a déjà travaillé plusieurs années à l'étranger, dans le Golfe, et il lui tarde de se marier, si possible avec une femme d'un niveau culturel et social égal au sien, et de même confession [...] il se renseigne auprès de son frère, établi depuis plusieurs années à Cincinnati, sur les procédures d'immigration aux États-Unis » (Rolin $2005: 44$ et 45).

7. Ainsi cette « inquiétude » de Sunny Paul « [...] au sujet des performances d'Ass-Marine, dont le retard était en train de prendre des proportions alarmantes [...] on sent qu'il envisage aussi comme une mise en cause de ses compétences personnelles et de celles de ses compatriotes » (Rolin 2005 : 47). Ou encore, après la faillite de la société les employant à Saint-Nazaire, cette réaction recueillie auprès de certains des travailleurs indiens mis à pied: «[...] qui semblent ébahis qu'un syndicat [la CGT, en l'occurrence] déploie une telle ardeur pour les soutenir, que la presse et une partie de l'opinion publique s'émeuvent de leur sort, et, par-dessus tout, qu'un tribunal puisse le cas échéant statuer en leur faveur » (Rolin 2005 : 47).

8. «[...] nous avons croisé sur le marché MM. Jeddy et Sharma [...] le premier musulman et le second hindou, et M. Jeddy a observé que "s'il y avait trois choses que l'on pouvait faire n'importe où en France, c'était boire, fumer et embrasser une fille”. Pendant le déjeuner [...] un autre convive, hindou celui-là, M. Jaggadish, est revenu sur le sujet, soulignant à quel point il 
était choqué de voir fumer des collégiennes dans la rue, et ironisant d'autre part sur le peu de fidèles qui se réunissaient le dimanche dans les églises » (Rolin 2005 : 47)

9. «En fin d'après-midi, le hall de la gare est envahi d'Indiens trempés par la pluie, fébriles, encadrés par quelques militants de la CGT qui négocient avec le personnel des guichets la gratuité de leur transport jusqu'à Nantes [...] le rassemblement, encadré par deux ou trois représentants de la CGT et quelques militants associatifs, reste tout d'abord assez grêle, apparemment parce que "les gars de Pornic" - ceux des Indiens qui sont logés dans un village de vacances de cette localité, distante d'une vingtaine de kilomètres - n'ont pas trouvé de moyen de transport pour le rejoindre [...] "Pour eux, vingt bornes c'est rien", commente avec désinvolture un employé français de l'entreprise italo-indienne en faillite, et mal lui en prend, car il se fait aussitôt rappeler à l'ordre par une militante associative au nom de la "civilisation millénaire", de la "non-violence", de "Gandhi", comme si tout cela était incompatible avec la marche à pied. » (Rolin 2005 : 51-52)

10. On peut évoquer, à propos d'une telle fragilisation, les analyses d'Anthony Giddens (2000 : 181-208, notamment) concernant la pluralisation des codes identitaires dans le contexte posttraditionnel de ce qu'il désigne du terme de high modernity.

11. Voir tout particulièrement le premier chapitre de l'étude que Bakhtine ([1975] 2006) consacre aux « Formes et temps du chronotope».

12. Voir, dans une perspective plus résolument pédagogique, Alain Lallemand (2011).

13. « En passant à hauteur de la gare maritime, j'ai observé en contrebas de la route un groupe de clandestins africains que des flics raccompagnaient vers la sortie de ce dispositif [...]. Par luimême [...] le conteneur est un objet assez anodin, plutôt laid - en dépit des couleurs brillantes dont le revêtent certains de ses utilisateurs [...]. À 20 heures tandis qu'au fond de la salle, sur un écran de grande taille, la chaîne Euronews présente des images horrifiantes de l'attentat de Madrid, au premier plan les Indiens menacés de licenciement jouent au billard sans leur accorder ne serait-ce qu'un regard » (Rolin $2005: 136,164$ et 216 respectivement).

14. Les études de Tim Cresswell (2006) et John Urry (2007) font ici référence. On consultera également, pour un aperçu plus synoptique, l'ouvrage de Peter Adey (2010).

15. On consultera également l'étude de Vincent Kaufmann (2002) pour prendre la mesure de mobilités s'affirmant comme pratiques sociales dans le contexte contemporain. Danilo Martuccelli et Anne Barrère (2005) proposent par ailleurs une étude tout à fait éclairante sur l'inscription des enjeux de mobilité dans la production littéraire contemporaine, en France tout du moins.

16. «Bien que, personnellement, je ne trépigne pas contre les lois sur l'immigration, dans la mesure où elles me paraissent nécessaires, je ressentais évidemment une certaine gêne à voyager si facilement, pour le plaisir et pour un prix très modique, à bord de ce navire spacieux et presque vide, alors que les clandestins devaient affronter de tels périls et acquitter de telles sommes pour voyager à fond de cale, sans aucune certitude d'arriver à bon port. » (Rolin 2005 : 148)

17. «[...] je n'éprouve aucune difficulté à envisager cette révolution du transport maritime sur un mode épique, même si je doute de ma capacité à faire partager ce point de vue. Pourtant, quoi de plus romanesque que le duel, mobilisant sur toutes les mers du globe des dizaines de navires et des centaines d'hommes [...] qui dans les années quatre-vingt opposa Malcolm McLean, l'Américain généralement crédité de l'invention du conteneur [...] à "Chairman" Chang, le Taïwanais parti de rien (comme il se doit), pour la maîtrise de cette innovation que constituaient alors les services “tour du monde" par porte-conteneurs. » (Rolin 2005 : 163)

18. «En fin de matinée, je me suis présenté à la médiathèque où je n'ai pas trouvé Miloud. Peutêtre était-ce parfois délibérément qu'il donnait de fausses indications sur son emploi du temps, afin de se draper de mystère ou de se rendre insaisissable. Peut-être ses "affaires" étaient-elles à 
l'origine de ce flou. Peut-être n'avait-il jamais dormi, contrairement à ce qu'il racontait, dans un wagon Corail stationné sur une voie de garage. » (Rolin 2005 : 139)

19. «Dans cette chambre que James partage avec le soudeur, une demi-douzaine d'Indiens se trouve rassemblée [...] pour assister à la présentation d'une cassette vidéo enregistrée quelques jours auparavant à l'occasion d'un repas pris en commun dans la même chambre [...]. Puis le silence se fait, et, sur l'écran, James interprète en solo, avec un léger accompagnement de casseroles et d'autres percussions domestiques, des airs mélancoliques du Karnataka. L'œil embué, il explique qu'il s'agit d'une chanson d'amour [...] destinée à sa femme, et d'une berceuse qu'il chantait autrefois à sa fille pour l'endormir. Et qu'il lui chante encore, précise-t-il, chaque fois qu'elle en fait la demande, à l'occasion de son coup de téléphone hebdomadaire aux ÉtatsUnis. » (Rolin 2005 : 56-57)

20. "Sur un quai de l'entrepôt Paul-Devot, une demi-douzaine de militants associatifs décharge d'une camionnette de la nourriture en vrac, puis dresse une table devant laquelle s'alignent peu à peu quelques dizaines de clandestins venus de plusieurs directions. » (Rolin 2005 : 125)

21. Le modèle des scapes que propose Arjun Appadurai (1996) tout comme sa théorisation des mobilités disjonctives ne sont pas sans résonnance narrative ici.

22. «[...] la situation matérielle des Indiens se détériore de jour en jour : depuis que les Chantiers ont rompu le contrat qui les liait à l'entreprise de climatisation, celle-ci, menacée d'une faillite imminente, a cessé de payer ses employés, lesquels n'ont pas perçu leur salaire du mois d'août. Dans l'attente d'une décision du tribunal de commerce, nul ne sait qui s'acquittera désormais des frais de logement ou de nourriture des Indiens, même si la mairie et le Secours populaire y pourvoient momentanément. » (Rolin $2005: 50)$

23. «Ce 19 mars 2009, la manifestation grossit toujours, mais sans colère, sans slogan réel, comme si sa seule revendication était sa propre masse. Gigantesque, démesurée, la foule a même fini par engloutir ceux qui l'avaient convoquée, les syndicats. On distingue parfois un chant [...]. Le cortège ne semble plus porté ni par les certitudes, ni par les doléances, mais traversé seulement de questions, qui volent de groupe en groupe : “Combien on est? Qu'est-ce qu'on va faire? Jusqu'à quand ça va durer ? Où on va ?" " (Aubenas $2010: 56$ )

24. "Victoria vient d'un tout autre pays, un village dans la Manche [...] À l'époque, la promenade $\mathrm{du}$ soir au village consistait à regarder passer le train rapide Paris-Caen, près de la gare de chemin de fer. [...] Victoria entre à la JOC [Jeunesse ouvrière chrétienne]. On la présente à quelques filles qui sont employées dans les foyers démunis et débordés. Victoria se dit que c'est pour elle. Elle se voit un avenir : elle aussi sera dans le social, et elle partira enfin du village [...]. Victoria peine à expliquer comment elle se retrouve à Caen. "On ne se posait pas tellement de questions à l'époque. On suivait la ligne de chemin de fer depuis nos villages, on descendait dans une grande ville." " (Aubenas $2010: 60$ et 61)

25. «En 1991, à l'occasion d'un reportage dans l'adrar des Iforas, au nord du Mali, ayant lié conversation avec le chef en second d'un camp de dissidents touaregs, et évoqué devant lui les années de jeunesse de mon père [...] il s'avéra presque sans aucun doute, par la coïncidence exacte des dates et des lieux, que son propre père était le sous-officier qui avait accompagné le mien dans ses méharées. » (Rolin 2005 : 197)

26. «[...] ruines d'une maison dont il semble qu'elle n'ait jamais été achevée, et qui est connue localement sous le nom de "maison de la folle" ou de "maison du pendu". Les recherches que j'ai entreprises à son sujet, tant auprès du Port autonome, sur le territoire duquel elle est bâtie, que de la mairie de Loon-Plage, dont elle dépend administrativement, ne m'ont pas permis d'en apprendre davantage. » (Rolin $2005: 64$ )

27. «Sans doute est-ce le lendemain, dimanche 12 octobre, que j'ai lu dans La Voix du Nord l'article concernant le BCMO, sa disparition programmée [...] Ou peut-être est-ce par hasard qu'en empruntant une fois de plus la chaussée des Darses, ce dimanche, je suis tombé sur le petit 
bâtiment rouge, à l'enseigne de Jokelson, où des artistes s'étaient installés, et que l'un d'eux m'a entretenu des menaces pesant sur le BCMO.» (Rolin 2005 : 75)

28. Giddens (1991), ainsi que les analyses ultérieures regroupées dans Runaway World (2000). Pour la problématisation des discours d'expertise telle qu'elle concerne la socialisation des situations de risque, voir tout particulièrement les travaux d'Ulrich Beck (2003).

29. On consultera l'étude de Christine Delory-Momberger (2009) pour une synthèse élargissant sensiblement l'approche des travaux sociologiques thématisant l'inscription sociale du récit de soi.

30. «Marilou et son homme habitent Caen : ils ont mis presque une heure avec leurs gilets fluorescents et leur tout petit scooter à lutter contre les bourrasques pour parcourir les quinze kilomètres de route. Sans nous connaître davantage, nous nous jetons dans les bras l'une de l'autre. Oui, nous ferons du covoiturage ensemble. » (Aubenas, $2010: 75$ )

31. «Marilou n'a ni permis ni voiture, mais on la croirait élevée pour survivre sur un parking d'hypermarché. De loin, et à l'oreille, elle reconnaît plus facilement une voiture que n'importe quel être humain, elle est capable de réciter les marques et les prix de tous les concessionnaires de la ville, sait déjà l'auto qu'elle achètera et avec quelles options - quand elle aura des sous, c'est-à-dire très bientôt. » (Aubenas, $2010:$ 76)

32. «Marilou a reçu une lettre, qu'elle n'a pas bien comprise. Il en ressortait qu'elle devait aller s'expliquer au siège de Youpi-Métal, à Lisieux. Son homme a haussé les épaules. Il ne sait pas lire. Lisieux, en train, ça fait cher, et en scooter ça fait loin. Le rendez-vous tombait à 9 heures, ce qui ne lui laissait pas le temps de finir son premier boulot. Toute l'affaire lui paraissait hors de prix et compliquée. » (Aubenas, $2010: 82$ )

33. Lire notamment Bruno Blanckeman (2005).

34. Voir les analyses de Dominique Viart (2005 : 235-251) quant à cette mouvance du littéraire vers l'écriture journalistique.

\section{RÉSUMÉS}

Isolant au sein de la pensée sociologique contemporaine les enjeux de mobilité et de réflexivité narrative, la présente étude se penche sur la façon dont les textualise le journalisme littéraire. Attentive à la production du récit social dans Le Quai de Ouistreham de Florence Aubenas (2010) et Terminal Frigo de Jean Rolin (2005), elle se propose de déterminer comment l'intelligibilité narrative de ce sous-genre du discours de presse intègre, dans le travail de figuration du social qui lui revient, des autorécits de mobilité problématisant l'unité et la composition du référent social.

This paper focuses on the representation of mobility and self-reflexivity in French literary journalism. It aims to articulate how these two sets of issues central to contemporary sociology shift social narratives in this sub-genre of non-fiction writing. The analysis compares to this end the literary reportage Le Quai de Ouistreham penned by French journalist Florence Aubenas (2010) and Jean Rolin's Terminal Frigo (2005). 
INDEX

Mots-clés : mobilité, réflexivité, journalisme littéraire, modernité avancée, récit de soi

Keywords : mobility, reflexivity, literary journalism, late modernity, self-narrative

\section{AUTEUR}

PASCAL GIN

Carleton University, Ottawa (Canada) 